\title{
Coverage by Randomly Deployed Wireless Sensor Networks
}

\author{
Peng-Jun Wan* Chih-Wei Yi ${ }^{\dagger}$
}

\begin{abstract}
One of the main applications of wireless sensor networks is to provide proper coverage of their deployment regions. $A$ wireless sensor network $k$-covers its deployment region if every point in its deployment region is within the coverage ranges of at least $k$ sensors. In this paper, we assume that the sensors are deployed as either a Poisson point process or a uniform point process in a square or disk region, and study how the probability of the $k$-coverage changes with the sensing radius or the number of sensors. Our results take the complicated boundary effect into account, rather than avoiding it by assuming the toroidal metric as done in the literature.
\end{abstract}

\section{Introduction}

One of the main applications of wireless sensor networks is to provide proper coverage of their deployment regions. Typically, the sensing range of a sensor is a (closed or open) circular disk centered at the sensor, whose radius is termed as the sensing radius of the sensor. For any positive integer $k$, a point is said to be $k$-covered by a sensor network if it falls in the sensing ranges of at least $k$ sensors, and a region is said to be $k$-covered if each point in this region is $k$-covered. In this paper, we study how the probability of a deployment region being $k$-covered by randomly deployed sensors changes with the sensing radius or the number of sensors. A precise description of the problems is given below.

Let $X_{1}, X_{2}, \cdots$ be independent and uniformly distributed random points on a bounded region $A$ in the plane. Given a positive integer $n$, the point process $\left\{X_{1}, X_{2}, \cdots, X_{n}\right\}$ is referred to as the uniform n-point process on $A$, and is denoted by $\mathcal{X}_{n}(A)$. Given a positive number $\lambda$, let $\operatorname{Po}(\lambda)$ be a Poisson random variable with

\footnotetext{
*Department of Computer Science, Illinois Institute of Technology, Chicago, IL 60616; and Department of Computer Science, City University of Hong Kong. Email: wan@cs.iit.edu.

$\dagger$ Department of Computer Science, Illinois Institute of Technology, Chicago, IL 60616; and Department of Computer and Information Science, National Chiao Tung University, Taiwan. Email: yichihw@iit.edu.
}

parameter $\lambda$, independent of $\left\{X_{1}, X_{2}, \cdots\right\}$. Then the point process $\left\{X_{1}, X_{2}, \cdots, X_{P o(\lambda)}\right\}$ is referred to as the Poisson point process with mean $\lambda$ on $A$, and is denoted by $\mathcal{P}_{\lambda}(A)$. Let $k$ be a fixed nonnegative integer, and $\Omega$ be the unit-area square or disk centered at the origin $o$. For any real number $t$, use $t \Omega$ to denote the set $\{t x: x \in \Omega\}$, i.e., the square or disk of area $t^{2}$ centered at the origin. Let $C_{n, r}$ (respectively, $\left.C_{n, r}^{\prime}\right)$ denote the event that $\Omega$ is $(k+1)$-covered by the (open or closed) disks of radius $r$ centered at the points in $\mathcal{P}_{n}(\Omega)$ (respectively, $\mathcal{X}_{n}(\Omega)$ ). Let $K_{s, n}$ (respectively, $\left.K_{s, n}^{\prime}\right)$ denote the event that $\sqrt{s} \Omega$ is $(k+1)$-covered by the unit-area (closed or open) disks centered at the points in $\mathcal{P}_{n}(\sqrt{s} \Omega)$ (respectively, $\mathcal{X}_{n}(\sqrt{s} \Omega)$ ). Then, we would like to study the asymptotics of $\operatorname{Pr}\left[C_{n, r}\right]$ and $\operatorname{Pr}\left[C_{n, r}^{\prime}\right]$ as $n$ approaches infinity, and the asymptotics of $\operatorname{Pr}\left[K_{s, n}\right]$ and $\operatorname{Pr}\left[K_{s, n}^{\prime}\right]$ as $s$ approaches infinity.

Let $\eta$ denote the peripheral of $\Omega$, which is equal to 4 (respectively, $2 \sqrt{\pi}$ ) if $\Omega$ is a square (respectively, disk). For any $\xi \in \mathbb{R}$, let

$$
\alpha(\xi)= \begin{cases}\frac{\left(\frac{\sqrt{\pi} \eta}{2}+e^{-\frac{\xi}{2}}\right)^{2}}{16\left(2 \sqrt{\pi} \eta+e^{-\frac{\xi}{2}}\right)} e^{-\frac{\xi}{2}}, & \text { if } k=0 \\ \frac{\sqrt{\pi} \eta}{2^{k+6}(k+2) !} e^{-\frac{\xi}{2}}, & \text { if } k \geq 1 .\end{cases}
$$

and

$$
\beta(\xi)= \begin{cases}4 e^{-\xi}+2\left(\sqrt{\pi}+\frac{1}{\sqrt{\pi}}\right) \eta e^{-\frac{\xi}{2}}, & \text { if } k=0 ; \\ \frac{\sqrt{\pi}+\frac{1}{\sqrt{\pi}}}{2^{k-1} k !} \eta e^{-\frac{\xi}{2}}, & \text { if } k \geq 1 .\end{cases}
$$

The mains results of this paper are summarized in the following two theorems.

$$
\begin{aligned}
& \text { Theorem } 1 \text { Let } r_{n}=\sqrt{\frac{\ln n+(2 k+1) \ln \ln n+\xi_{n}}{\pi n}} \\
& \lim _{n \rightarrow \infty} \xi_{n}=\xi \text { for some } \xi \in \mathbb{R} \text {, then } \\
& 1-\beta(\xi) \leq \lim _{n \rightarrow \infty} \operatorname{Pr}\left[C_{n, r_{n}}\right] \leq \frac{1}{1+\alpha(\xi)}, \text { and } \\
& 1-\beta(\xi) \leq \lim _{n \rightarrow \infty} \operatorname{Pr}\left[C_{n, r_{n}}^{\prime}\right] \leq \frac{1}{1+\alpha(\xi)} . \\
& \text { If } \lim _{n \rightarrow \infty} \xi_{n}=\infty \text { then } \\
& \lim _{n \rightarrow \infty} \operatorname{Pr}\left[C_{n, r_{n}}\right]=\lim _{n \rightarrow \infty} \operatorname{Pr}\left[C_{n, r_{n}}^{\prime}\right]=1 .
\end{aligned}
$$




$$
\begin{aligned}
& \text { If } \lim _{n \rightarrow \infty} \xi_{n}=-\infty \text {,then } \\
& \quad \lim _{n \rightarrow \infty} \operatorname{Pr}\left[C_{n, r_{n}}\right]=\lim _{n \rightarrow \infty} \operatorname{Pr}\left[C_{n, r_{n}}^{\prime}\right]=0 .
\end{aligned}
$$

Theorem 2 Let $\mu(s)=\ln s+2(k+1) \ln \ln s+\xi(s)$. If $\lim _{s \rightarrow \infty} \xi(s)=\xi$ for some $\xi \in \mathbb{R}$, then

$$
\begin{aligned}
& 1-\beta(\xi) \leq \lim _{s \rightarrow \infty} \operatorname{Pr}\left[K_{s, \mu(s) s}\right] \leq \frac{1}{1+\alpha(\xi)}, \text { and } \\
& 1-\beta(\xi) \leq \lim _{s \rightarrow \infty} \operatorname{Pr}\left[K_{s, \mu(s) s}^{\prime}\right] \leq \frac{1}{1+\alpha(\xi)} .
\end{aligned}
$$

If $\lim _{s \rightarrow \infty} \xi(s)=\infty$, then

$$
\lim _{s \rightarrow \infty} \operatorname{Pr}\left[K_{s, \mu(s) s}\right]=\lim _{s \rightarrow \infty} \operatorname{Pr}\left[K_{s, \mu(s) s}^{\prime}\right]=1 .
$$

If $\lim _{s \rightarrow \infty} \xi(s)=-\infty$, then

$$
\lim _{s \rightarrow \infty} \operatorname{Pr}\left[K_{s, \mu(s) s}\right]=\lim _{s \rightarrow \infty} \operatorname{Pr}\left[K_{s, \mu(s) s}^{\prime}\right]=0 .
$$

The equalities (3) and (4) will be derived from the inequalities (1) and (2) by a perturbation argument. The inequality (2) will be obtained from the inequality (1) by a de-Poissonization argument. Theorem 2 can be proved from Theorem 1 by using a scaling argument. Due to the limitation on paper length, we will skip the detail and only the proof of the equality (1) will be given in this paper.

We remark that the probabilistic studies of $k$-coverage by a random point process have been conducted for $k=1$ in [1] and arbitrary integer-valued constant $k$ in [5] but with certain limitations. Both studies assume Poisson point processes on a square and use the toroidal metric, rather than the Euclidean metric which is more relevant to the applications. This renders their results hardly applicable to wireless sensor networks. Indeed, the smallest sensing radius or sensor density to ensure the $k$-coverage under the toroidal metric almost surely fails to guarantee the $k$ coverage under the Euclidean metric. The assumption of the toroidal metric technically eliminates the boundary effect under the Euclidean metric.

In what follows, $\|x\|$ is the Euclidean norm of a point $x \in \mathbb{R}^{2}$, and $|A|$ is shorthand for 2-dimensional Lebesgue measure (or area) of a measurable set $A \subset \mathbb{R}^{2}$. All integrals considered will be Lebesgue integrals. The topological boundary of a set $A \subset \mathbb{R}^{2}$ is denoted by $\partial A$. The (closed or open) disk of radius $r$ centered at $x$ is denoted by $D_{r}(x)$. For any $x, y \in \Omega$, let

$$
\begin{aligned}
v_{r}(x) & =\left|D_{r}(x) \cap \Omega\right|, \\
v_{r}(y \backslash x) & =\left|\left(D_{r}(y) \backslash D_{r}(x)\right) \cap \Omega\right|, \\
\phi_{n, r}(x) & =\sum_{i=0}^{k} \frac{\left(n v_{r}(x)\right)^{i}}{i !} e^{-n v_{r}(x)}, \\
\phi_{n, r}(y \backslash x) & =\sum_{i=0}^{k} \frac{\left(n v_{r}(y \backslash x)\right)^{i}}{i !} e^{-n v_{r}(y \backslash x) .}
\end{aligned}
$$

An event is said to be asymptotic almost sure (abbreviated by a.a.s.) if it occurs with a probability converges to one as $n \rightarrow \infty$. The symbols $o, \sim, \gtrsim, \lesssim$ refer to either the limit $n \rightarrow \infty$. To avoid trivialities, we tacitly assume $n$ to be sufficiently large if necessary. For simplicity of notation, the subscripts will be frequently suppressed.

\section{Geometric Ingredients}

For any $r>0$ and a unit-area square $\Omega$, we partition $\Omega$ into three subregions $\Omega_{r}(0), \Omega_{r}(1)$ and $\Omega_{r}(2)$ as illustrated in Figure 1(a): $\Omega_{r}(0)$ consists of all points in $\Omega$ apart from the sides of $\Omega$ by at least $r, \Omega_{r}$ (1) consists of all points in $\Omega$ apart from some side of $\Omega$ by less than $r$ and from all other sides by at least $3 r$, and $\Omega_{r}(2)$ consists of the rest points in $\Omega$. For any $x \in \Omega_{r}(i), v_{r}(x) \geq 2^{-i} \pi r^{2}$.

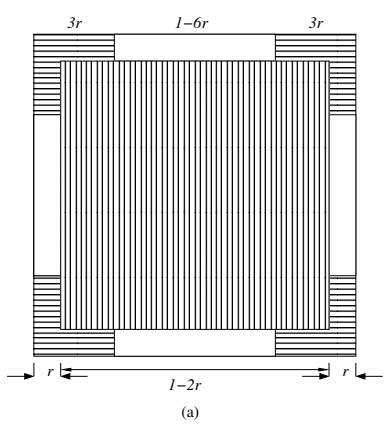

Figure 1. Parition of $\Omega:(a) \Omega$ is square, (b) $\Omega$ is disk.

For any $r>0$ and a unit-area disk $\Omega$, we partition $\Omega$ into three subregions $\Omega_{r}(0), \Omega_{r}(1)$ and $\Omega_{r}(2)$ as illustrated in Figure 1(b): $\Omega_{r}(0)$ is the disk of radius $\frac{1}{\sqrt{\pi}}-r$ centered at $\mathbf{o} ; \Omega_{r}(1)$ is the annulus of radii $\frac{1}{\sqrt{\pi}}-r$ and $\sqrt{\frac{1}{\pi}-r^{2}}$ centered at $\mathbf{o}$; and $\Omega_{r}(2)$ is the annulus of radii $\sqrt{\frac{1}{\pi}-r^{2}}$ and $\frac{1}{\sqrt{\pi}}$ centered at $\mathbf{o}$. For any $x \in \Omega_{r}(i), v_{r}(x) \geq 2^{-i} \pi r^{2}$.

In the remaining of this section, we introduce the following lemma. Due to the limitation on paper length, its proof is not given here.

Lemma 3 Let $S$ be a disk of radius $s$. For any $r>s$, let $\digamma$ be the set of points $(x, y) \in \mathbb{R}^{2} \times \mathbb{R}^{2}$ satisfying that there exists $z \in S$ such that $\|x-z\|=\|y-z\|=r$ and $\overrightarrow{x y} \times$ $\overrightarrow{x z} \geq 0$. Then the Lebesgue measure of $\digamma$ is $\left(4 \pi r^{2}\right)|S|$.

\section{Critical Sensing Radius}

This section is devoted to the proof of the equality (1). The inequality (1) consists of the asymptotic upper bound and the asymptotic lower bound on $\operatorname{Pr}\left[C_{n, r_{n}}\right]$. 
The proof of the asymptotic upper bound on $\operatorname{Pr}\left[C_{n, r_{n}}\right]$ is based on the observation that the event $V_{n, r}$ implies that the $(k+1)$-vacancy $V_{n, r}$ defined by the Lebesgue measure (i.e., area) of the set of points in $\Omega$ which are not $(k+1)$ covered by the closed disks of radius $r$ centered at the points in $\mathcal{P}_{n}(\Omega)$ is zero. Hence, $\operatorname{Pr}\left[C_{n, r}\right]$ is upper bounded by $\operatorname{Pr}\left[V_{n, r}=0\right]$. Based on the Cauchy-Schwartz inequality, $\operatorname{Pr}\left[V_{n, r}=0\right]$ can be further upper bounded in terms of the mean and variance of $V_{n, r}$.

The proof of the asymptotic lower bound on $\operatorname{Pr}\left[C_{n, r_{n}}\right]$ is based on the following characterization of $(k+1)$-coverage by open disks: Let $L_{n, r}$ denote the number of $(n, r)$ crossing points where an $(n, r)$-crossing point is either an intersection point of $\partial \Omega$ and $\partial D_{r}(X)$ for some $X \in$ $\mathcal{P}_{n}(\Omega)$, or an intersection point of $\partial D_{r}(X)$ and $\partial D_{r}(Y)$ for some $X, Y \in \mathcal{P}_{n}(\Omega)$ respectively, and $M_{n, r}$ denote the number of $(n, r)$-crossing points which are not $(k+1)$ covered by the open disks of radius $r$ centered at the points in $\mathcal{P}_{n}(\Omega)$. Then, $C_{n, r}$ occurs if and only if $L_{n, r}>0$ and $M_{n, r}=0$ (see, e.g., [3]). Hence,

$$
\begin{aligned}
\operatorname{Pr}\left[C_{n, r}\right] & =\operatorname{Pr}\left[L_{n, r}>0, M_{n, r}=0\right] \\
& =\operatorname{Pr}\left[L_{n, r}>0\right]-\operatorname{Pr}\left[L_{n, r}>0, M_{n, r}>0\right] \\
& =\operatorname{Pr}\left[L_{n, r}>0\right]-\operatorname{Pr}\left[M_{n, r}>0\right] \\
& \geq \operatorname{Pr}\left[L_{n, r}>0\right]-E\left[M_{n, r}\right],
\end{aligned}
$$

where the last inequality follows from the Markov inequality.

\subsection{Mean and Variance of the $(k+1)-$ Vacancy}

The following lemma gives the asymptotic mean of the $(k+1)$-vacancy and an asymptotic upper bound on the variance of the $(k+1)$-vacancy.

Lemma 4 Let $r_{n}=\sqrt{\frac{\ln n+(2 k+1) \ln \ln n+\xi_{n}}{\pi n}}$ with $\lim \xi_{n}=$ $\xi$ for some $\xi \in \mathbb{R}$. Then,

$$
E\left[n\left(n \pi r_{n}^{2}\right) V_{n, r_{n}}\right] \sim \begin{cases}e^{-\xi}+\frac{\sqrt{\pi} \eta}{2} e^{-\frac{\xi}{2}} & \text { if } k=0, \\ \frac{\sqrt{\pi} \eta}{2^{k+1} k !} e^{-\frac{\xi}{2}} & \text { if } k \geq 1\end{cases}
$$

and

$\operatorname{Var}\left[n\left(n \pi r_{n}^{2}\right) V_{n, r_{n}}\right] \lesssim \begin{cases}16 e^{-\xi}+32 \sqrt{\pi} \eta e^{-\frac{\xi}{2}} & \text { if } k=0 \\ \frac{(k+1)(k+2) \sqrt{\pi} \eta}{2^{k-4} k !} e^{-\frac{\xi}{2}} & \text { if } k \geq 1 .\end{cases}$

\subsection{Crossing Points}

In this subsection, we prove the following lemma.

Lemma 5 Let $r_{n}=\sqrt{\frac{\ln n+(2 k+1) \ln \ln n+\xi_{n}}{\pi n}}$ with $\lim \xi_{n}=$ $\xi$ for some $\xi \in \mathbb{R}$. Then, $L_{n, r_{n}}>0$ is asymptotically almost sure and $E\left[M_{n, r_{n}}\right] \lesssim \beta(\xi)$.

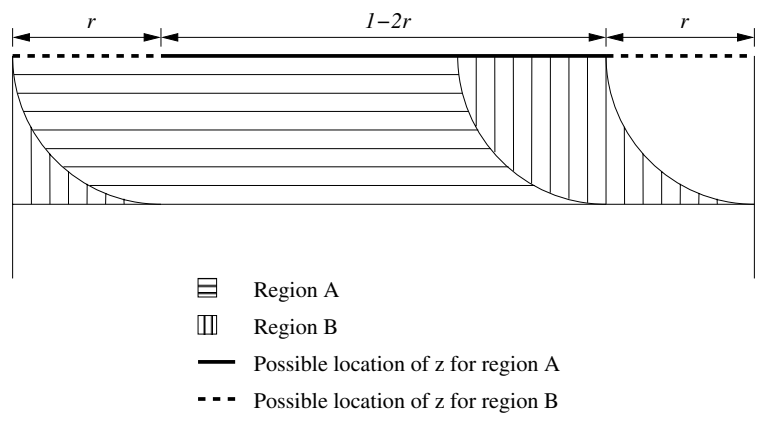

Figure 2. Region $A$ and $B$ for the crosspoint in $\partial \Omega$.

For simplicity, we suppress the subscripts. An $(n, r)$ cross pointing is said to be of the first (respectively, second) type if it is an intersection point between $\partial \Omega$ and $\partial D(X)$ for some $X \in \mathcal{P}_{n}(\Omega)$ (respectively, between $\partial D(X)$ and $\partial D(Y)$ for some $X, Y \in \mathcal{P}_{n}(\Omega)$ ). Let $L^{\prime}$ be the number of crossing points of the first type. Then,

$$
\begin{aligned}
\operatorname{Pr}[L=0] & \leq \operatorname{Pr}\left[L^{\prime}=0\right]=e^{-n|\Omega \backslash \Omega(0)|}=e^{-\Theta(n r)} \\
& =o(1) .
\end{aligned}
$$

Thus, $L>0$ is asymptotically almost sure.

Let $M^{\prime}$ (respectively, $M^{\prime \prime}$ ) be the number of crossing points of the first (respectively, second) type which are not $(k+1)$-covered. By Lemma 4, $E\left[M_{n, r_{n}}\right] \lesssim \beta(\xi)$ would follow from

$$
E\left[M^{\prime}\right] \sim \frac{\eta e^{-\frac{\xi}{2}}}{k ! 2^{k-1} \sqrt{\pi}} \text { and } E\left[M^{\prime \prime}\right] \leq 4 E\left[n\left(n \pi r^{2}\right) V\right] .
$$

We begin with the limit of $E\left[M^{\prime}\right]$.

First we consider the case that $\Omega$ is a square region. Let $X$ be a random point and $Q$ be the event that the right halfcircle of $\partial D(X)$ and the upper side of $\Omega$ intersects at a point which is not $(k+1)$-covered. By symmetry,

$$
E\left(M^{\prime}\right)=8 n \operatorname{Pr}(Q)
$$

Let $A$ be the set of points of $x$ such that the right half-circle of $\partial D(x)$ and the upper side of $\Omega$ intersects at a point which is at a distance of at least $r$ from both vertical sides of $\Omega$, and $B$ be the set of points of $x$ such that the right half-circle of $\partial D(x)$ and the upper side of $\Omega$ intersects at a point which is at a distance of less $r$ from either the left vertical side of $\Omega$ or the right vertical side of $\Omega$ (see figure 2). Then,

$$
\operatorname{Pr}(Q)=\int_{A \cup B} \operatorname{Pr}(Q \mid X=x) d x .
$$


After some calculation, we have

$$
\begin{aligned}
& n \int_{A} \operatorname{Pr}(Q \mid X=x) d x \sim \frac{1}{k ! 2^{k} \sqrt{\pi}} e^{-\frac{\xi}{2}}, \text { and } \\
& n \int_{B} \operatorname{Pr}(Q \mid X=x) d x=o(1) .
\end{aligned}
$$

Therefore,

$$
E\left(M^{\prime}\right)=8 n \operatorname{Pr}(Q) \sim \frac{\eta e^{-\frac{\xi}{2}}}{k ! 2^{k-1} \sqrt{\pi}} .
$$

Now we consider the case that $\Omega$ is a disk region. Each node in $\Omega \backslash \Omega(0)$ produces two crossing points of the first type. Each node on $\partial \Omega(0)$ produces exactly one crossing pint of the first type, but $\partial \Omega(0)$ has zero measure. All other nodes does not produce any crossing point of the first type. For any point $z \in \partial \Omega$, since $\phi_{n, r}(z) \sim$ $\frac{1}{k !}\left(\frac{n \pi r^{2}}{2}\right)^{k} e^{-\frac{n \pi r^{2}}{2}}$, we have

$$
\begin{aligned}
E\left(M^{\prime}\right) & \sim 2 n|\Omega \backslash \Omega(0)| \frac{1}{k !}\left(\frac{n \pi r^{2}}{2}\right)^{k} e^{-\frac{n \pi r^{2}}{2}} \\
& \sim \frac{\eta e^{-\frac{\xi}{2}}}{k ! 2^{k-1} \sqrt{\pi}} .
\end{aligned}
$$

Next we derive the asymptotic upper bound on $E\left[M^{\prime \prime}\right]$. Fix an ordered pair of random nodes $X$ and $Y$. Let $Z$ be the intersecting point of the two circles $\partial D(X)$ and $\partial D(Y)$ with $\overrightarrow{X Y} \times \overrightarrow{X Z} \geq 0$. Let $Q$ denote the event that $Z$ lies inside $\Omega$ and $Z$ is not $(k+1)$-covered. Then by symmetry,

$$
E\left[M^{\prime \prime}\right]=n(n-1) \operatorname{Pr}[Q] .
$$

By Lemma 3, the probability that $Z$ lies in a sufficiently small circular disk of area $d z$ centered at a point $z \in \Omega$ is at most $4 \pi r^{2} d z$. Thus,

$$
\begin{aligned}
E\left[M^{\prime \prime}\right] & \leq n(n-1) \int_{\Omega} \phi_{n, r}(z) 4 \pi r^{2} d z \\
& \leq 4 E\left[n\left(n \pi r^{2}\right) V\right] .
\end{aligned}
$$

\subsection{Proof of Theorem 1}

Here we prove the inequality (1). For simplicity, we suppress the subscripts. By Lemma 4, it is straightforward to show that

$$
\frac{E\left[n\left(n \pi r^{2}\right) V\right]^{2}}{\operatorname{Var}\left[n\left(n \pi r^{2}\right) V\right]} \gtrsim \alpha(\xi) \text {. }
$$

By the Cauchy-Schwartz inequality,

$$
\begin{aligned}
\operatorname{Pr}[V=0] & \leq \frac{\operatorname{Var}[V]}{E\left[V^{2}\right]}=\frac{1}{1+\frac{E[V]^{2}}{\operatorname{Var}[V]}} \\
& =\frac{1}{1+\frac{E\left[n\left(n \pi r^{2}\right) V\right]^{2}}{\operatorname{Var}\left[n\left(n \pi r^{2}\right) V\right]}} \lesssim \frac{1}{1+\alpha(\xi)} .
\end{aligned}
$$

Thus,

$$
\operatorname{Pr}[C] \leq \operatorname{Pr}[V=0] \lesssim \frac{1}{1+\alpha(\xi)} .
$$

On the other hand, by Lemma 5,

$$
\operatorname{Pr}[C] \geq \operatorname{Pr}\left[L_{n, r}>0\right]-E\left[M_{n, r}\right] \gtrsim 1-\beta(\xi) .
$$

So, the inequality (1) holds.

\section{Conclusion}

We address the asymptotic $(k+1)$-coverage of a square or disk region by a Poisson or uniform point process. A major technical challenge is the handling of the boundary effect. For the purpose of comparison between with and without the boundary effect, let us consider the asymptotic $(k+1)$-coverage of a square by Poisson point process with unit-area coverage range. With boundary effect, the asymptotic $(k+1)$-coverage requires that the sensor density $n / s$ should grow with the area $s$ at least according to

$$
n / s=\ln s+2(k+1) \ln \ln s+\xi(s)
$$

with $\lim _{s \rightarrow \infty} \xi(s)=\infty$. Without the boundary effect, the asymptotic $(k+1)$-coverage only requires that the sensor density $n / s$ grows with the area $s$ according to

$$
n / s=\ln s+(k+2) \ln \ln s+\xi(s)
$$

with $\lim _{s \rightarrow \infty} \xi(s)=\infty$ [5].

\section{References}

[1] P. Hall. Introduction to the Theory of Coverage Processes. John Wiley \& Sons, 1988.

[2] P.-J. Wan and C.-W. Yi. Asymptotic critical transmission radius and critical neighbor number for $k$-connectivity in wireless ad hoc networks. In Proceedings of the 5th ACM International Symposium on Mobile Ad Hoc Networking and Computing, pages 1-8, May 24-26 2004.

[3] X. Wang, G. Xing, Y. Zhang, C. Lu, R. Pless, and C. Gill. Integrated coverage and connectivity configuration in wireless sensor networks. In Proceedings of the first international conference on Embedded networked sensor systems, pages 2839. ACM Press, November 2003.

[4] C.-W. Yi, P.-J. Wan, X.-Y. Li, and O. Frieder. Asymptotic distribution of the number of isolated nodes in wireless ad hoc networks with bernoulli nodes. In IEEE Wireless Communications and Networking Conference, March 16-20 2003.

[5] H. Zhang and J. Hou. On deriving the upper bound of $\alpha$ lifetime for large sensor networks. In Proceedings of the 5th ACM International Symposium on Mobile Ad Hoc Networking and Computing, pages 121-132, May 24-26 2004. 